\title{
Propuesta para un vocabulario de especialidad en biología reproductiva de las plantas*
}

\author{
ELENA RAIMÚNDEZ-URRUTIA \\ Departamento de Biología de Organismos, División de Ciencias Biológicas, \\ Universidad Simón Bolívar (Caracas, Venezuela)
}

\section{INTRODUCCIÓN}

Hablar sobre un área específica de conocimiento científico-técnico implica utilizar un léxico que, en mayor o menor proporción, se distingue del de la llamada lengua común por el uso de términos con significado propio y específico que definen el dominio sobre el cual se aplica dicho conocimiento (Cabré, 2003; Santamaría Pérez, 2006). Los términos han sido definidos como las unidades que designan los conceptos propios de cada especialidad, y se considera que no difieren mucho de las palabras de la lengua común en el aspecto formal, pero sí en cuanto a criterios pragmáticos y comunicativos (Cabré, 2003; Cordero Monge, 2009). Se habla así de lengua de especialidad, que es el conjunto de los términos usados y compartidos por un grupo de hablantes en el desarrollo de una actividad especializada (Cabré, 2003; Santamaría Pérez, 2006). El carácter especializado de los términos está determinado, principalmente, por la temática sobre la que se trata en el dominio del área de conocimiento científico-técnico específico en que se usan, pero también por el tipo de interlocutores (emisores y receptores) que comparten esa información especializada, por las circunstancias comunicativas en las que se produce, y por el propósito mismo de la comunicación (Cabré, 1993 y 2003).

Un área de conocimiento científico-técnico en biología que ha alcanzado gran importancia en los últimos años es el de la biología reproductiva de las plantas, que tradicionalmente se venía considerando como parte de la botánica, pero que hoy día puede definirse como un área de especialidad por sí misma debido al aumento en tiempo reciente de los estudios relacionados con ella (Ramírez, 2008). Además, en la actualidad, las investigaciones en esta área no se limitan solo a generar conocimiento per se de las características reproductivas de las plantas y de sus interacciones ecológicas, sino que sus resultados se utilizan para tomar decisiones en otras áreas de investigación — por ejemplo, relacionadas con la restauración de áreas degradadas, con el manejo agronó-

* Este trabajo ha sido posible gracias a la asesoría constante del Dr. José-Álvaro Porto Dapena, quien pacientemente ayudó a darle forma a la idea inicial. Igualmente, el financiamiento otorgado por la Universidad Simón Bolívar permitió la estancia de la autora en la Universidade da Coruña durante su año sabático. 
mico de especies de interés y con la conservación de la biodiversidad (Traveset, 2002; Ramírez, 2008) - , por lo que aumenta cada vez más el número de investigadores que genera información al respecto y el público interesado en obtenerla y comprenderla.

Para la difusión de los resultados de las investigaciones en la biología reproductiva de las plantas se utiliza una terminología específica, relacionada con las propias estructuras reproductivas - flores/frutos, óvulos/semillas - , con los métodos de investigación sobre los fenómenos y procesos que se desarrollan, y con las interacciones que establecen con factores bióticos y abióticos del ambiente durante esta fase de su ciclo de vida. La mayoría de los textos se producen y publican en inglés, pero existe una comunidad creciente de especialistas en los países de habla hispana que, en ocasiones, deben producir y publicar sus textos en español; sin embargo, y a pesar de la creciente importancia de estas investigaciones en los países hispanohablantes, su terminología no ha sido recogida como léxico de especialidad en ninguna obra terminológica en español. El Diccionario de botánica de Pius Font Quer, que ha sido usado desde su aparición en 1953 como referencia obligada en los estudios botánicos en español, no recoge muchos de los términos, pues estos se han creado o se han incorporado del inglés durante el auge de los estudios en biología reproductiva de plantas de los últimos 30-40 años. Recientemente se han elaborado algunas listas de términos relacionados con la reproducción en las plantas, pero exclusivamente para explicar los vocablos utilizados en una obra particular, por lo que su alcance es muy limitado; también pueden conseguirse listas más extensas que están disponibles en la Red — lo que facilita su acceso-, pero no se conoce si han sido validadas por especialistas.

Se hace necesario, entonces, el desarrollo de un vocabulario de especialidad en la biología reproductiva de las plantas, en el que se presenten definiciones concretas de los conceptos representados por sus términos, acompañadas de descripciones que faciliten la comprensión de los textos que se producen en esta especialidad, y que contribuyan a la normalización del uso de dichos términos en español. Por esto, el objetivo principal de este trabajo ha sido elaborar una propuesta de vocabulario, en la que se defina la estructura que debe tener y sus características generales, de acuerdo a las propias características de los términos que se usan en esta especialidad. Para elaborar la propuesta ha sido necesaria la recopilación de un corpus textual del cual se han extraído los términos usados en esta disciplina, y con los cuales se han podido definir las características que debe tener el vocabulario y el modelo de estructura para la redacción de los artículos terminológicos, en el que se detallan los criterios formales y de fondo que se deben considerar. Como novedad con respecto al Diccionario de botánica y a las listas de términos mencionadas, en esta propuesta se ha considerado lo que ya viene siendo práctica común en otras obras terminológicas, que las definiciones y las descripciones estén basadas en la realización más concreta posible de los términos, que es su uso en los textos de especialidad en español; los textos producidos por especialistas y el conocimiento sobre el tema que posee el especialista que realiza el vocabulario pueden considerarse como autoridad y, por tanto, como sancionadores del uso de los términos (Porto Dapena, 2002). 


\section{FUENTES DOCUMENTALES Y ELABORACIÓN DEL CORPUS TEXTUAL PARA LA EXTRACCIÓN DE TÉRMINOS}

Para la elaboración del corpus textual se ha hecho una búsqueda a través de la Red con ayuda del motor de búsquedas Google, introduciendo las palabras clave: «biología reproductiva de plantas», «ecología reproductiva de plantas», «estrategia reproductiva de plantas», «reproducción en plantas», «polinización», «dispersión de semillas». Con esta búsqueda se han tratado de recuperar textos que cubriesen la fase reproductiva de las plantas, desde el inicio de la floración hasta lo que puede considerarse su última etapa, que es la dispersión e implantación de las semillas.

De las fuentes documentales así obtenidas se han seleccionado artículos científicos publicados en revistas especializadas de áreas como botánica, ecología, agronomía, ciencias forestales o biología tropical. También se han recuperado capítulos de libros, trabajos de grado (licenciatura y maestría), tesis doctorales, programas de cursos y asignaturas, y artículos solo disponibles en línea referidos a investigaciones o a comunicaciones formales sobre la biología reproductiva de las plantas. La mayor parte de estos documentos pueden ser considerados de especialización alta porque están dirigidos a otros especialistas del área, están publicados en los medios propios de difusión de esta área de especialidad, y su finalidad es la de dar a conocer los resultados de las investigaciones realizadas por los especialistas (Cabré, 2007); sin embargo, algunos documentos podrían considerarse de especialización media porque su finalidad es la divulgación de conceptos, pero siempre destinados a unos receptores más o menos involucrados con el tema.

El corpus textual en el que se ha basado la propuesta reúne así 178 documentos producidos en el período comprendido entre 1985 y 2011, provenientes tanto de España como de buena parte de los países hispanoamericanos, por lo que puede considerarse suficiente para obtener una muestra representativa de los términos utilizados en esta especialidad en el ámbito hispanohablante; este corpus está conformado por cerca de 1.445.135 palabras.

\section{EXTRACCIÓN Y SELECCIÓN DE TÉRMINOS}

El trabajo de extracción y selección de los términos se ha hecho de forma manual asistida por el ordenador. Se ha utilizado el programa ExtPhr32 (2006, de Timothy C. Craven) para extraer del corpus tanto las llamadas palabras gráficas (conjunto de letras unidas entre sí y separadas de otras letras que las anteceden y otras que las siguen por espacios en blanco, Porto Dapena, 2002) como los sintagmas terminológicos y las unidades fraseológicas (conjunto de palabras que se unen para realizar de forma unitaria una misma función sintáctica dotada de sentido, Cabré, 1993) —en adelante se hará referencia a estos dos últimos como términos sintagmáticos-; este programa se ha considerado adecuado para la extracción de los términos de este pequeño corpus puesto que permite obtener de forma fácil una lista de los términos que aparecen en los textos con su frecuencia de aparición. 
Con los resultados obtenidos se ha hecho una selección manual para identificar los términos (simples y sintagmáticos) asociados al estudio de la biología reproductiva de las plantas. Esta selección se ha basado principalmente en dos criterios: uno subjetivo, que ha tomado en cuenta el conocimiento de la especialidad, al considerar el uso específico de estos términos en el estudio de la reproducción en las plantas; y uno objetivo, que ha considerado la frecuencia con la que estos términos aparecieron en el corpus utilizado; este segundo criterio permitirá, principalmente, tomar decisiones sobre términos sinónimos para ver cuál de ellos se usa preferentemente y que se escogerá como lema del artículo terminológico. Lo que se ha tomado en cuenta ha sido que los términos aparecieran en más de un texto y, preferiblemente, en textos de autores diferentes para evitar, en lo posible, el sesgo en la forma del término y en su definición. Finalmente, para la redacción de los artículos terminológicos que aquí se presentan como ejemplos, se han seleccionado citas de los términos escogidos y del contexto que los acompaña en los documentos del corpus textual; se ha seleccionado una cantidad de contexto que se ha considerado suficiente para entender el uso del término y su definición.

\section{ESTRUCTURA DEL VOCABULARIO}

La estructura que se propone para el Vocabulario de la biología reproductiva de las plantas (en adelante VBRP) está basada en el modelo propuesto para el Diccionario 'Coruña' de la lengua española actual (Porto Dapena et al., 2007), con las modificaciones necesarias para una obra terminológica y no lingüística, y de mucha menor envergadura.

\subsection{Características generales del vocabulario}

El VBRP será monolingüe español, aunque para cada término se dará su equivalente en inglés, ya que es la lengua de publicación y difusión más usada en los textos de esta especialidad. Tendrá también carácter sincrónico al incorporar únicamente documentos producidos en los últimos 30-40 años, que se considera el período de auge de los trabajos en la biología reproductiva de las plantas, incluyendo los realizados en español, y en los cuales se han creado la mayor parte de los términos. Se tratará de unificar el uso de algunos términos para los cuales exista confusión en cuanto a su significado (homonimia/polisemia), y se tratará también de unificar el uso de términos sinónimos o de variantes gráficas; como ya se ha señalado, los documentos en español que pueden recuperarse de la Red provienen tanto de España como de buena parte de los países hispanoamericanos, por lo que se puede considerar que los términos seleccionados deben ser los más estándares en lengua española para esta especialidad.

Los artículos terminológicos seguirán un enfoque semasiológico, es decir, se irá desde el significante hasta la idea o significado y, paralelamente, en otras ocasiones, del signo a la cosa; tanto la definición como la información descriptiva que acompañarán a los términos serán autorizados con citas de textos reales producidos por los especialistas y tomadas del corpus textual. Las definiciones del vocabulario serán, de acuerdo a estos criterios, de tipo enciclopédico, en el sentido de que aportarán información relevante sobre las realidades que se estudian en esta especialidad, que pueda ser 
obtenida a partir de los textos seleccionados, pero también hará un aporte lexicográfico, al definir los conceptos y fijar los términos correspondientes.

Por último, en cuanto al soporte, se quiere que el vocabulario esté disponible en formato digital, dados los avances tecnológicos alcanzados en cuanto a los equipos para la lectura de documentos electrónicos y a los programas para su manejo, que podrán facilitar su consulta en línea o su consulta en un soporte electrónico. El formato y la tipografía que se usen irán de acuerdo a las características del sitio web en el que se publique $y$, aunque se siga un enfoque semasiológico, al tratarse de formato digital, no será necesaria la ordenación alfabética de los artículos terminológicos. Las características sugeridas para cada elemento de los artículos terminológicos se señalan en la descripción de la microestructura.

\subsection{Características de los términos y delimitación del dominio de la especialidad}

Los términos que se van a incluir en el vocabulario han sido extraídos del corpus textual elaborado con los documentos producidos por los especialistas en la biología reproductiva de las plantas. En este sentido se trata de términos cultos, de registro formal, pues son los utilizados por estos especialistas para producir los textos, tanto orales como escritos, con los que comunican los resultados de sus investigaciones en los espacios formales para ello, como son revistas, libros especializados, trabajos académicos y reuniones científicas, y para lo cual deben elaborar el discurso de una manera consciente y reflexiva.

Por otra parte, los términos que se incluyan deben aparecer con una frecuencia de uso tal que refleje su aceptación, al menos, por una parte de la comunidad de especialistas del área en el ámbito hispanohablante. En caso de comprobarse que el uso de algún término se limita solo a los especialistas de una nacionalidad o de una región, se considerará como una variante gráfica o como un sinónimo - dependiendo del casoen cuyos casos se incluirán como parte del enunciado - en el caso de la variante- o como subentrada - en el caso del sinónimo- del término que tenga mayor frecuencia de uso en cuanto a extensión geográfica en el área de influencia del español, tal como se explicará en la descripción de la microestructura de los artículos terminológicos, aunque también se incluirá como entrada que remita al término más frecuente; la información sobre el país o región de uso se indicará a continuación del enunciado o en la subentrada del término más restringido.

Se tratará, como es de esperar, de un vocabulario selectivo, que incluirá solo términos aplicados, si no exclusivamente a las plantas, sí concretamente al estudio de lo que se conoce como su biología reproductiva - desde el inicio de la floración hasta la dispersión e implantación de las semillas-, aunque esta pueda tener límites difusos y, en ocasiones, se entremezclen y utilicen términos que podrían ser considerados también de otras áreas de especialidad biológica, como la genética —al considerar la base genética que determina las características reproductivas de las plantas (p. ej. autoincompatibilidad - — o la ecología — al considerar todas las interacciones bióticas y abióticas que establecen las plantas para su reproducción (p. ej. mutualismo)—. Sin embargo, esto no 
desvirtúa en forma alguna la naturaleza concreta y específica de la terminología de esta especialidad, sino que la complementa. Por otra parte, algunos de los términos podrían ser considerados de la lengua común (p. ej. abortar), pero serán incluidos cuando representen un aspecto o rasgo definido y concreto en el estudio reproductivo de las plantas. Finalmente, no se va a incluir la nomenclatura propia de los grupos de plantas consideradas angiospermas ni de los grupos de animales con los que establecen relaciones.

La última consideración con respecto a las características de las unidades terminológicas tiene que ver con su tipo gramatical. En general, en las terminologías se incluyen principalmente sustantivos, adjetivos y verbos, o unidades sintagmáticas que actúen como tales, debido a la naturaleza de las cosas que definen. En cualquiera de los casos puede tratarse, como ya se ha dicho, de términos simples (palabras gráficas) o de términos sintagmáticos (incluyendo unidades fraseológicas) que, como unidad, cumplan alguna de estas funciones, y serán los que se incluyan en el vocabulario.

\subsection{Macroestructura del vocabulario}

\subsubsection{Criterios para establecer las entradas}

\subsubsection{Unidades simples y unidades sintagmáticas}

A diferencia del procedimiento habitual utilizado en los diccionarios semasiológicos que considera únicamente vocablos simples, en el caso de este vocabulario de especialidad se incluirán entradas representadas tanto por unidades simples (palabras gráficas, p. ej. autogamia) como por unidades sintagmáticas (grupos de palabras que unidas constituyen un término dotado de sentido en biología reproductiva, p. ej. éxito reproductivo, síndrome de dispersión) ya que, en general, estas últimas se refieren a estructuras, fenómenos, procesos o interacciones bien definidos y con significado propio dentro de la biología reproductiva de las plantas. Estas unidades sintagmáticas aparecerán como una única entrada, de acuerdo a la primera palabra que las forme que, en general, suele corresponder a sustantivos.

autogamia. $f$. Sistema de apareamiento en el que se puede dar la unión de un gameto masculino...

éxito reproductivo. $m$. Proporción de semillas viables producidas al final de un ciclo reproductivo por unidad reproductiva...

síndrome de dispersión. $m$. Conjunto de características morfológicas, fisiológicas y de comportamiento de las diásporas...

Sin embargo, existen otros casos de unidades sintagmáticas cuyo significado corresponde a una ampliación específica del significado de uso de una unidad simple ( $\mathrm{p}$. ej. dispersión secundaria como ampliación de dispersión) o de otra unidad sintagmática (p. ej. sistema de autoincompatibilidad esporofitico y sistema de autoincompatibilidad gametofitico como ampliación de sistema de autoincompatibilidad), en cuyo caso estos sintagmas más amplios serán considerados como subentradas dentro de la entrada correspondiente a la unidad cuyo significado amplían, lo que será explicado en la mi- 
croestructura; estas unidades sintagmáticas aparecerán también como entradas, pero remitiendo a la unidad en cuyo artículo se estudian.

dispersión. $f$. Separación de las diásporas de la planta madre... $\| \sim$ secundaria. $f$. La que ocurre con posterioridad a la llegada de la diáspora al suelo una vez...

dispersión secundaria. $f$. V. dispersión.

sistema de autoincompatibilidad. $m$. Conjunto de características, principalmente genéticas, pero muchas veces asociadas a...

$\| \sim$ esporofítico. $m$. El determinado por la interacción entre el genoma diploide de la cubierta del grano de polen y el genoma diploide del estigma-estilo...

$\| \sim$ gametofítico. $m$. El determinado por la interacción entre el genoma haploide del grano de polen y el genoma diploide del estigma-estilo...

sistema de autoincompatibilidad esporofítico. $m$. V. sistema de autoincompatibilidad.

sistema de autoincompatibilidad gametofítico. $m$. V. sistema de autoincompatibilidad.

\subsubsection{Categorías gramaticales}

Con respecto a la categoría gramatical, la mayoría de los términos parecen corresponder a sustantivos, lo que caracteriza a las lenguas de especialidad (Ahumada, 2001). En los documentos revisados sobre la biología reproductiva de las plantas, los sustantivos se usan para definir no solo objetos o entidades, sino también relaciones y procesos, restándole valor al uso, sobre todo, de los verbos; al parecer, son muy pocos los verbos que se usan en estos textos con un sentido restringido de especialidad y la mayor parte pertenecen a la lengua común. Pese a ello, existen algunos verbos que pueden considerarse términos específicos de la biología reproductiva de las plantas (p. ej. polinizar), a partir de los cuales se han derivado otros términos sustantivos (p. ej. polinización, polinizador), también con significado en la especialidad. Por último, algunos términos corresponden a adjetivos que indican una relación de pertenencia a o de cualidad de (p. ej. monoico, -ca). Se considerarán, entonces, tres tipos de entradas con respecto a la categoría gramatical: las correspondientes a verbos, las correspondientes a sustantivos y las correspondientes a adjetivos.

\subsubsection{Variantes y términos sinónimos}

En caso de presentarse variantes de un término en cuanto a su escritura (p. ej. enantiostilia enantioestilia), el artículo terminológico se desarrollará en la entrada correspondiente a la variante que se haya registrado con mayor frecuencia de uso en el corpus textual. Los términos menos frecuentes serán incluidos también como entrada, pero haciendo remisión al término más frecuente, en cuya entrada aparecerán como parte del enunciado, tal como se explicará en la microestructura. Si el criterio de frecuencia no fuera lo suficientemente discriminante para seleccionar el término que incluirá el artículo terminológico, se utilizará un criterio alfabético, desarrollando la definición en la entrada que alfabéticamente debería aparecer antes y el otro término remitirá al prime- 
ro. En el caso de términos sinónimos (p. ej. antesis apertura floral), el menos frecuente se incluirá como subentrada en la entrada correspondiente al más frecuente e, igualmente, aparecerá como entrada haciendo remisión al sinónimo más frecuente.

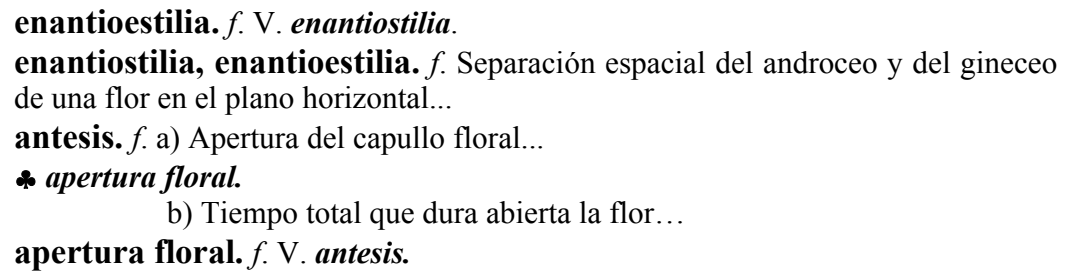

\subsubsection{Homónimos y términos polisémicos}

No se han identificado casos de homonimia y muy pocos de polisemia entre los términos, lo que parece ser una característica general en las terminologías usadas en las áreas de especialidad, ya que en estas los términos se crean en función de realidades concretas que deben ser designadas y a las cuales se les suelen asignar términos con un único significado muy preciso (Santamaría Pérez, 2006; Cordero Monge, 2009). Los términos homónimos se consideran gráficamente iguales pero etimológica o gramaticalmente diferentes - en este segundo caso, solo cuando una de las formas del término homónimo es verbal y la otra sustantiva o adjetival- (Porto Dapena et al., 2007); en el caso de identificarse términos homónimos, estos se considerarán como entradas independientes, cada una acompañada de su correspondiente artículo terminológico. Por su parte, los términos polisémicos se consideran términos con diferente significado pero con un significante de un único origen etimológico (Porto Dapena et al., 2007); en estos casos, cada uno de los significados será considerado una acepción o una subacepción y serán tratados todos dentro de una sola entrada, tal como se describirá en la microestructura de los artículos terminológicos.

\subsubsection{Ordenación de las entradas}

A pesar de seguirse un enfoque semasiológico y, en ese sentido, partir de los términos al significado, no es necesario definir una ordenación alfabética de las entradas debido al formato digital que se propone para el VBRP. Solo en el caso de encontrarse términos homónimos deberán aparecer en una sola búsqueda, en la que la ordenación se hará de acuerdo a criterios gramaticales (primero la forma sustantiva o la adjetiva y luego la verbal), cronológicos (de acuerdo a su antigüedad de uso) si pertenecen a la misma categoría gramatical y, finalmente, de frecuencia de uso, en caso de no poder resolver el orden con los dos criterios anteriores. Las subentradas estarán ubicadas después de las acepciones o subacepciones, en el orden que se definirá en la microestructura de los artículos terminológicos. 


\subsection{Microestructura del vocabulario}

\subsubsection{El enunciado}

En todas las entradas, tanto de las que remitan a otro término como de las que constituyan un artículo terminológico, el enunciado presentará una de estas tres posibles formas:

1) Estará formado solo por el término, simple o sintagmático, en su forma base, es decir, como lema, cuando se trate de una unidad terminológica correspondiente a un sustantivo que no presente formas flexivas de género (p. ej. dioecia o banco de semillas), o cuando corresponda a un verbo, que aparecerá en infinitivo (p. ej. emascular).

banco de semillas. $m$. a) Cantidad de semillas viables en estado de latencia que se encuentran generalmente enterradas en el suelo y...

b) Muestra de semillas conservadas ex situ en condiciones...

banco de semillas del suelo. $m$. V. banco de semillas.

dioecia. $f$. Sistema sexual en el que unos individuos de la población poseen solo flores masculinas y los otros solo flores femeninas...

emascular. $v$. Remover de forma mecánica el androceo de la flor.

2) Estará formado por alguna forma de flexión (de género o de número) del término, cuando esta sea la única forma en la que aparece en el corpus textual (p. ej. adicógama), con lo que se entiende que es la única forma de uso en la biología reproductiva de las plantas.

adicógama. adj. Se dice de la flor en la que la maduración del gineceo y la del androceo ocurren simultáneamente...

3) Estará formado por el lema del término, seguido de la indicación de la flexión femenina, cuando tanto la forma masculina (forma no marcada) como la femenina aparezcan usadas en el corpus textual (p. ej. xenógamo, -ma). En este último caso, la terminación de la flexión femenina irá precedida de la última consonante del lema (si la última sílaba es consonántica), que irá, a su vez, precedida de guion, todo separado del lema por una coma; en caso de que la forma masculina y/o la femenina aparezcan en el corpus tanto en singular como en plural, el lema y la terminación femenina se escribirán en singular.

xenógamo, ma. adj. Se dice de la especie de planta o del sistema de apareamiento en los que se promueve la fecundación de los óvulos con polen proveniente de...

Las variantes en la escritura de algunos términos se incluirán como parte del enunciado, separadas por una coma del término más frecuente que origina la entrada (p. ej. agamospérmico, -ca, agamospermo, -ma). En el enunciado de los términos homónimos - si se consiguieran - se agregará un superíndice escrito en números arábigos y se mantendrá el mismo formato de letra ya descrito.

agamospérmico, -ca, agamospermo, -ma. adj. Que tiene la capacidad de producir semillas sin que ocurra la fusión de gametos masculinos y femeninos. Propio o correspondiente a la agamospermia. (V. agamospermia). 
agamospermo, -ma. adj. V. agamospérmico.

El enunciado se escribirá en minúscula, negrita y en un cuerpo de letra mayor al resto de los textos que lo acompañen, y estará separado de estos por un punto.

\subsubsection{La remisión a otro término}

La remisión a otro término podrá hacerse desde entradas correspondientes a términos que representen una variante o un sinónimo, en las que se remitirá a la entrada de la variante o del sinónimo de uso más frecuente, y desde entradas que correspondan a términos sintagmáticos que amplíen el significado de un término simple, en las que se remitirá a la entrada del término simple. Estas entradas estarán formadas por el enunciado, seguido de la marca gramatical que corresponda al término - como se explica más adelante - y acompañado por la abreviatura V. (por véase), seguida del término al que se remite, el cual irá escrito en negrita y cursiva, pero en un tamaño de letra menor al enunciado - igual al tamaño del texto del artículo terminológico-.

Se usará, además, otra forma de remisión, que se hará a términos que se consideren relacionados con el término que constituye el artículo terminológico. Para indicarla, también se utilizará la abreviatura $\mathrm{V}$. antecediendo al término relacionado al cual se remite. Este tipo de remisión podrá aparecer al final de la definición de un término, entre paréntesis, remitiendo a los términos que aparezcan en la propia definición y que estén recogidos en el vocabulario; o como subentrada, al final del artículo y antes de la subentrada del término en inglés — como se explicará más adelante- remitiendo a otros términos relacionados que no se usen en la definición pero que también estén recogidos en el vocabulario.

\subsubsection{La marcación}

Se considera que en este vocabulario serán necesarias, en forma general, solo marcas gramaticales $\mathrm{y}$, de uso específico en algunos términos, marcas diatópicas, tal como se explicará a continuación.

Las marcas gramaticales que se usarán estarán referidas a las categorías de sustantivo -indicada con las abreviaturas para las subcategorías: $m$. por masculino (p. ej. cociente polen-óvulo) y $f$. por femenino (p. ej. carga de polen)—, adjetivo (adj.) (p. ej. dicógama) y verbo (v.) (p. ej. dispersar), tanto para marcar términos simples como sintagmáticos; estas marcas se colocarán inmediatamente después del enunciado del artículo, escritas en un cuerpo de letra menor a este, pero igual al resto del texto del artículo, en cursiva y sin negrita. Las marcas gramaticales aparecerán en todas las entradas. Finalmente, solo se incluirá marca gramatical en las acepciones si la categoría gramatical difiere entre ellas y la misma consideración se hará en el caso de las subacepciones.

carga de polen. $f$. Cantidad de granos de polen depositados en un área específica, bien sea sobre el cuerpo de los visitantes florales o bien... 
cociente polen/óvulo. $m$. Número de granos de polen producido por cada óvulo en una flor. Se considera un buen estimador...

dicógama. adj. Dicho de una flor que presenta un desfase en la maduración del gineceo y del androceo. Se dice también de una especie con...

dispersar. $v$. Remover y transportar las diásporas maduras desde la planta madre hasta una nueva ubicación...

La variación diatópica o geográfica que pudiera identificarse se marcará colocando el nombre del país o de la región en la entrada del término que se use de forma restringida; sin embargo, no se especificarán abreviaturas ya que se considera que serán pocos los casos en los que haya de indicarse esta variación; esta marcación se colocará entre corchetes, en una letra de menor tamaño que el enunciado - igual al tamaño del texto del artículo terminológico- en negrita y cursiva, y antes de la abreviatura que indica la remisión, puesto que, en general, se tratará de variantes de frecuencia de uso baja que remitirán al término sinónimo o variante más frecuente.

No se considera necesario el uso de ningún otro tipo de marcación en este vocabulario. No se requiere marcación diastrática, diafásica ni connotativa porque, como ya se señaló, se trata de términos cultos utilizados en el ámbito hispanohablante en los medios formales para la comunicación de los resultados que se producen sobre la reproducción de las plantas. Tampoco se requiere marcación diacrónica, porque todos los documentos corresponden a un solo período sincrónico. Finalmente, tampoco se usarán marcas de frecuencia de uso, porque esta ya quedará reflejada con el tipo de entrada, puesto que los usos más frecuentes generarán entradas de artículos terminológicos y los menos frecuentes, remisiones.

\subsubsection{El cuerpo del artículo}

\subsubsection{Forma de las entradas}

A continuación del enunciado y de la marca de la categoría gramatical a la que corresponda el término se colocará la definición, cuando el término tenga una sola acepción, o se colocarán las definiciones de las diferentes acepciones que tenga el término, numeradas consecutivamente con números arábigos, o de las diferentes subacepciones, introducidas con letras minúsculas en orden alfabético, seguidas de paréntesis de cierre. Para considerar acepciones diferentes de un término, cada una de ellas debe referirse a diferentes paradigmas léxicos (Porto Dapena et al., 2007), esto es, que en cada una de las acepciones indicadas el término se use con un significado concreto y diferente al resto de las acepciones, con hiperónimos, cohipónimos, hipónimos, etc., diferentes; si, por el contrario, pueden hacerse definiciones particulares de un término, determinadas solo por el contexto, sin que cambie el significado del término (Porto Dapena et al., 2007), dichas definiciones se considerarán subacepciones. Obviamente, cada definición correspondiente a una acepción o a una subacepción irá autorizada con las citas de los textos extraídos del corpus en los cuales se pueda verificar el sentido indicado. 
anemocoria, anemocoría. $f$. a) Modo de dispersión de las diásporas llevada a cabo por el viento. Asociada a ambientes con pocas interacciones bióticas. Es uno de los más frecuentes en las plantas. (V. diáspora, dispersar).

Estos factores actúan en beneficio de la eficacia en el modo de dispersión (anemocoria) común de estos aquenios con vilano... (Monteagudo Picris 2009, 38). - De acuerdo a las características morfoanatómicas descritas, el principal sistema de dispersión de las tres especies estudiadas es la anemocoria... (Mendoza Estudio 2010, 46). - Las semillas son del tipo Epidendrum [...]; al mismo tiempo se observan características derivadas dentro de la subfamilia, como una testa más pronunciada que el embrión y endospermo ausente, resultando en una cavidad que se llena de aire facilitando la anemocoria (Ospina Brassia 2007, 92). - En plantas, la anemocoría, la exo- y endozoocoría y la talasocoría... (Traveset Islas 2009, 159).

b) Síndrome asociado a la dispersión de las diásporas por el viento, caracterizado por diásporas ligeras, en general de pequeño tamaño y con estructuras que facilitan el vuelo (alas, pelos). (V. diáspora, síndrome de dispersión, dispersar).

Este estudio evaluó los mecanismos de dispersión de Polygala vayredae analizando el funcionamiento de sus síndromes de dispersión (anemocoria y mirmecocoria, Fig. 3)... (Castro Polygala 2009, 81). — La anemocoria [...] suele ser común en semillas pequeñas... (Mendoza Estudio 2010, 6).

anemocoría. $f$. V. anemocoria.

Las definiciones serán la parte más importante del artículo terminológico porque constituyen el verdadero aporte del vocabulario, ya que su redacción se basará en la información contenida en los textos, apoyada en el conocimiento del especialista. Las definiciones serán, mayormente, mixtas en cuanto a su composición, porque incluirán el concepto que exprese el contenido significativo del término (en el ejemplo anterior, el concepto es: Modo de dispersión de las diásporas llevada a cabo por el viento), representado por las características esenciales de la realidad que definen, e incluirán una descripción de otras características no esenciales de dicha realidad y que complementen el concepto (en el ejemplo anterior, las características complementarias son: Asociada a ambientes con pocas interacciones bióticas. Es uno de los más frecuentes en las plantas).

La elaboración de las definiciones se hará siguiendo los criterios de adecuación general, adecuación específica dentro de un campo de especialidad, y adecuación en su forma de expresión (Cabré, 1993; Porto Dapena, 2002). Además, puesto que lo que se busca es hacer un vocabulario útil para los especialistas, estudiantes y aprendices de la biología reproductiva de las plantas, y para cualquier persona que requiera conocer alguno de sus términos, debe procurarse la sencillez, transparencia y simplicidad en la formulación de las definiciones, pero buscando que incluyan la información pertinente. Como ya se ha señalado, se deberá estar pendiente del uso en las definiciones de otros términos no incluidos en el vocabulario para no caer en pistas perdidas, o del uso del propio término para evitar los círculos viciosos (Cabré, 1993; Porto Dapena, 2002). En este sentido, todos los términos usados en las definiciones deberán corresponder a entradas del vocabulario; de no aparecer como tales por no haber sido encontrados en el corpus textual, se buscará un término sinónimo que corresponda, o se usará una forma descriptiva de los mismos. Finalmente, la definición irá escrita en letra redonda y cuerpo de letra menor al enunciado. 


\subsubsection{Forma de las subentradas}

Las subentradas se ubicarán dentro del artículo terminológico después de las citas que autoricen la definición siguiendo un orden definido.

- Primero, subentrada(s) correspondiente(s) al(los) sinónimo(s) del término de la entrada: estará(n) introducida(s) con el topo *, seguido del término sinónimo, en negrita y cursiva, y acompañado por una cita que autorice su uso.

- Segundo, subentrada en la que se remite a algún(os) término(s) relacionado(s): estará introducida por el topo , seguido de la abreviatura V. (véase) y de los términos a los que se remite, en cursiva y negrita.

- Tercero, subentrada en la que se indica el(los) equivalente(s) en inglés: estará introducida por el topo $\bullet$, seguido del término en inglés escrito en cursiva.

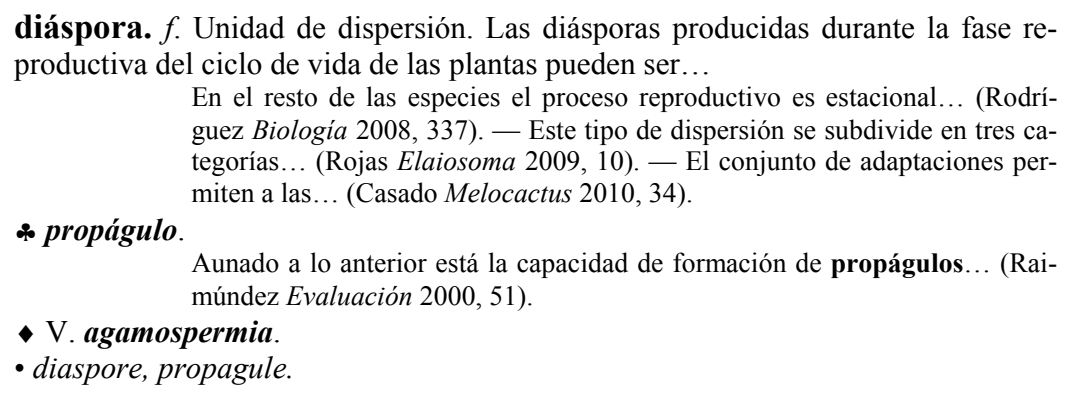

Todas estas subentradas se escribirán con un cuerpo de letra del mismo tamaño que el artículo terminológico, con sangrado simple con respecto al enunciado.

Algunos términos traducidos textualmente del inglés no corresponden con la forma aceptada en español (p. ej. aborción); sin embargo, por su aparición en los textos de biología reproductiva de las plantas, se indicarán como variantes o como sinónimos pero colocándoles el símbolo ${ }^{\otimes}$ para resaltar que no son de uso en español y aparecerán como entrada que remita al término que se propone como correcto.

aborción. $f$. V. aborto.

aborto, ${ }^{\otimes}$ aborción. $m$. Acción y efecto de abortar. En el caso de las flores o de los frutos, el aborto puede...

- Cuarto, subentrada(s) correspondiente(s) a el(los) término(s) sintagmático(s) que amplíen el significado de uso de un término simple y que lo incluyan al inicio: estará(n) introducida(s) por una doble pleca $(\|)$, seguida de virgulilla $(\sim)$ en sustitución del término simple y acompañados por la parte complementaria del término sintagmático. Todo esto constituirá el enunciado de este tipo de subentrada por lo que se escribirá en negrita, pero en cuerpo de letra igual al artículo terminológico, es decir, menor al cuerpo de letra del enunciado de la entrada. A continuación se colocará la definición correspondiente al término sintagmático, que se acompañará con las citas que lo autoricen; se usará una sangría doble para el enunciado, la definición y las citas de estas subentradas. 
hercogamia. $f$. Separación espacial del androceo y del gineceo en una misma flor. Puede darse tanto en el plano horizontal como...

En $Q$. schomburgkiana el estambre y el estilo están inclusos dentro de la corola y... (Rodríguez Biología 2008, 345). — La separación espacial entre sexos es considerada como... (Suárez Convolvulus 2004, 588). - El estudio de

- V. enantiostilia, heterostilia.

la biología floral mostró que... (Piña Opuntia 2007, 9).

- herkogamy.

$\|$ recíproca. Aquella en la que existe más de un morfo floral con respecto a la altura del androceo y del gineceo...

La enantiostilia, considerada como un tipo de hercogamia recíproca [...], en

E. uncinatum reduce la interferencia... (Rodríguez Aspectos 2004, 30). - La distilia es un polimorfismo caracterizado por la presencia de hercogamia recíproca... (Taisma Sistema 2005, 2). — Las especies diestílicas se caracterizan por presentar poblaciones... (Cuesta Palicourea 2008, v).

- V. distilia, enantiostilia, heterostilia.

- reciprocal herkogamy.

Debe tenerse presente que no tienen que aparecer todos los tipos de subentradas en un artículo terminológico, pero siempre se mantendrá este orden para los que aparezcan. Además, este orden de las subentradas se mantendrá para cada acepción o para cada subacepción después de las citas que las autoricen y en los casos que corresponda. Finalmente, en las subentradas que representen términos sintagmáticos pueden aparecer, a su vez, estas subentradas, estableciendo un segundo nivel de ordenación.

\subsubsection{Las citas y su fuente documental}

Como ya se ha señalado, cada una de las acepciones, subacepciones y subentradas de los artículos terminológicos estarán autorizadas por citas extraídas de los textos que conforman el corpus; se incluirá el número de citas necesario para respaldar la información incluida en la definición. La selección de las citas en las que se pueda verificar el contexto de uso de los términos se hará, entonces, con el criterio de texto mínimo, pero completo, de forma que contenga clara, pero escuetamente la información necesaria para la comprensión del significado del término. De esta forma, no podrán ser oraciones gramaticalmente incompletas, aunque deben evitarse los elementos superfluos, que podrán ser sustituidos por tres puntos entre corchetes ([...]), usados de acuerdo con las normas que indica la ortografía española. Los textos de las citas se iniciarán siempre con mayúscula aunque no aparezcan así en el original, pero se respetará la ortografía y la puntuación. Si para la mejor comprensión del texto se requiere añadir información sobreentendida que haya sido citada en alguna otra parte del documento, esta podrá agregarse escribiéndola entre corchetes. Las citas estarán escritas en cuerpo de letra menor al artículo terminológico, en párrafo aparte y con sangrado simple con respecto al enunciado. Además, se escribirán en forma continua, separadas por una raya larga (-).

síndrome de polinización. $m$. Conjunto de características morfológicas, fisiológicas y de comportamiento de las flores, asociadas a la polinización por un determinado agente biótico o abiótico. (V.polinizar).

En general, el síndrome de polinización por colibríes es muy parecido al síndrome de polinización por mariposas diurnas [...]. Las flores polinizadas 
por colibríes son predominantemente tubulares [...] y las polinizadas por mariposas diurnas pueden tener tubos largos y estrechos [...], tipo trompeta [...], la longevidad de las flores polinizadas por colibríes frecuentemente es mayor que un día [...] y en flores polinizadas por mariposas diurnas frecuentemente tienen una longevidad de un día [...], el volumen de néctar es considerablemente menor en especies polinizadas principalmente por mariposas [...] comparado con flores polinizadas por colibríes (Ramírez Llanos 2007, 403). - Las características morfológicas de las flores y otras como color, olor, antesis diurna y recompensa ofrecida corresponden con un síndrome de polinización por abejas, siendo los principales polinizadores especies de las familias Anthophoridae y Apidae (Rodríguez Aspectos 2004, 23). — Existe una creciente evidencia en plantas insulares, aunque también en continentales, de la incidencia de ambofilia (fenómeno que presentan las especies con síndrome de polinización entomófila pero que adoptan una combinación de polinización por viento y por insectos) (Traveset Islas 2009, 165).

- V. anemofilia, entomofilia, hidrofilia, ornitofilia, quiropterofilia.

- pollination syndrome.

Estas citas irán acompañadas de la abreviatura empleada para identificar a los documentos de los cuales se extrajeron, cuya información bibliográfica aparecerá desarrollada en la nómina de fuentes que se incluirá junto con el vocabulario. La abreviatura que acompañe a la cita (p. ej. Arceo Chamaecrista 2008, 13) estará compuesta por el primer apellido del autor $-\mathrm{o}$ del primer autor en casos de coautoría一, escrito en letra redonda; una palabra significativa del título del documento, escrita en letra cursiva; el año de publicación del documento — cuando este no se suministre se indicará SF (sin fecha) - y la página del documento en la que aparece la cita —cuando esté disponible-, estos dos últimos también en letra redonda; y todo entre paréntesis. En el caso de diferentes documentos de un mismo autor o de documentos de autores con el mismo apellido se tendrá el cuidado de que la palabra seleccionada del título sea diferente para cada documento.

\subsubsection{Elementos no textuales}

Al final de algunos artículos terminológicos se podrán incluir hipervínculos que lleven a esquemas, representaciones gráficas, fotos o direcciones web, y podrán incluirse, además, hipervínculos a vídeos para complementar la descripción dada con la definición para facilitar la comprensión del término.

dehiscencia. $f$. a) Apertura espontánea de las anteras para la liberación de los granos de polen o de los frutos para la liberación de las semillas.

Sus flores perfectas son capaces de autofertilizarse, ya que las piezas fértiles están dispuestas de forma tal que en el momento de la dehiscencia de las anteras es posible el contacto entre el polen y el estigma (Musicante Cologania 2008, 14). - Es probable, además, que la mayor oferta de polen también se produzca en las horas centrales del día, cuando el aumento de la temperatura y la disminución de la humedad atmosférica han provocado la dehiscencia de muchas anteras (Ortiz Ceratonia 1996, 545). — La dehiscencia de los frutos en el momento en que hay un aumento en las precipitaciones, permite que las semillas dispongan de agua en el suelo para germinar y establecerse durante el período de lluvias (Figueredo Agave 2011, 1366). - Elaeagia uxpanapensis fructificó en tres ocasiones, durante los cuatro años del presente estudio. De la primera fructificación fue posible apreciar los últimos frutos, ya en 
proceso de dehiscencia, al inicio del estudio, por tanto, fue una observación sólo [sic] parcial (Brenes Elaeagia 2001, 993).

b) Forma como se abren las anteras para la liberación de los granos de polen o los frutos para la liberación de las semillas. Puede ocurrir de diversas formas, relacionadas con el modo de polinización o el modo de dispersión (p. ej. en el sentido longitudinal de la antera o del fruto: $d$. longitudinal; a través de pequeños orificios o poros: $d$. foraminada; de forma violenta: $d$. explosiva) (V. dispersar, polinizar).

Cuando la flor acaba de abrir, uno o dos de los estambres se elongan y se abren las anteras por dehiscencia longitudinal. [...]. Poco a poco los estilos van creciendo, a medida que el resto de los estambres maduran y liberan el polen (Buide Silene 2000, 99). - Si bien no hay registros de los polinizadores de esta especie, la misma posee anteras con dehiscencia foraminada $[\ldots]$ y se conoce que en esos casos, la polinización se realiza por abejas hembras capaces de recoger el polen a través de la vibración de las anteras; este comportamiento se denomina "buzz pollination" o polinización por vibración (Cabrera Solanum 2010, 78). - Un total de ocho modos diferentes de dispersión de diásporas fueron considerados: i) viento, ii) agua, iii) viento combinado con agua, iv) balística (dehiscencia explosiva), v) granivocoria (hormigas y vertebrados), vi) aves, vii) aves y otros vertebrados, viii) mamíferos (epizoocoria y endozoocoria por mamíferos omnívoros y herbívoros) (Herre-

- dehiscence. ra Predicción 2009, 264).

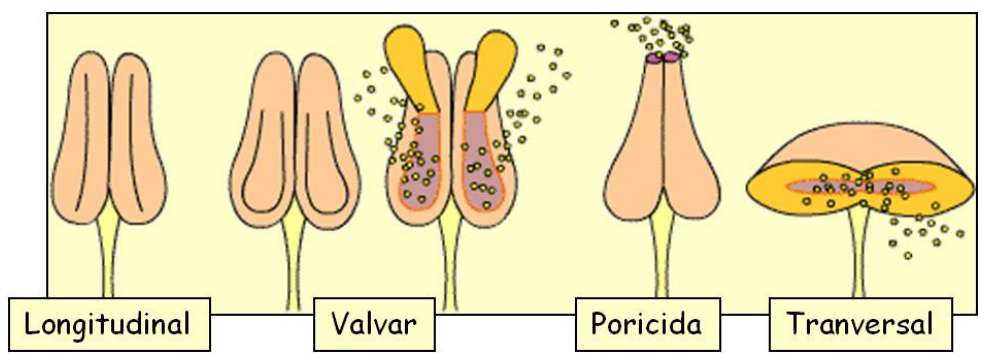

Representación gráfica de algunas formas de dehiscencia de las anteras para la liberación del polen (Tomado de http:/www.biologia.edu.ar/botanica/tema4/ 4_6androceo.htm).

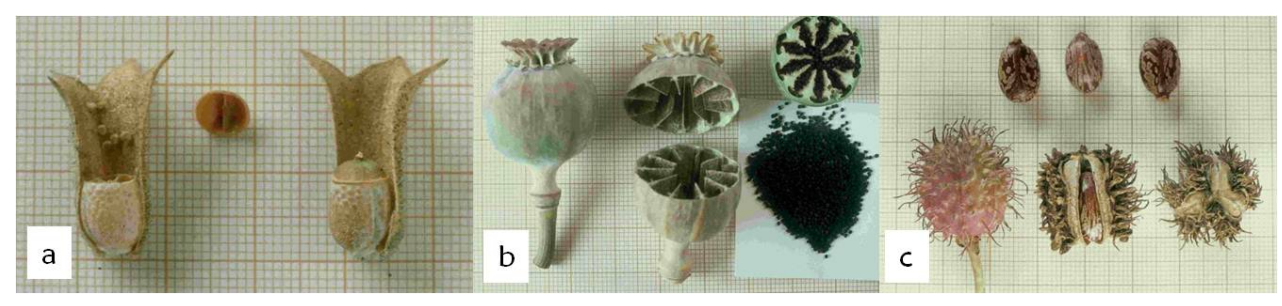

Algunas formas de dehiscencia de los frutos: a) transversal, b) foraminada y c) longitudinal (Tomado de http://www.almediam.org/Equipo_Filabres/Frutos/ pagina_003_4_frutos.htm). 


\section{REFERENCIAS BIBLIOGRÁFICAS}

Ahumada, Ignacio (2001): «Problemas de la definición enciclopédica en las palabras especializadas», en M. Bargalló, E. Forgas, C. Garriga, A. Rubio, J. Schnitzer, eds., Las lenguas de especialidad y su didáctica, Tarragona, Universitat Rovira i Virgili, pp. 59-68 [en línea en http://digital.csic.es/bitstream/10261/11525/1/Ahumada.pdf; consulta: $13 / 07 / 2011]$.

CABRÉ, M. TERESA (1993): La terminología. Teoría, metodología, aplicaciones, Barcelona, Antártida-Empúries.

(2003): «El lenguaje científico desde la terminología», en Jornadas sobre Aproximaciones al lenguaje de la ciencia, Burgos, Fundación Instituto Castellano y Leonés de la Lengua, pp. 19 [en línea en http://www.upf.edu/pdi/dtf/teresa.cabre/docums/ ca03lgci.pdf; consulta: 13/07/2011].

(2007): «Constituir un corpus de textos de especialidad: condiciones y posibilidades», en M. Ballard y C. Pineira-Tresmontant, eds., Les corpus en linguistique et en traductologie, Arras, Artois Presses Université, pp. 89-106 [en línea en http://www.upf.edu/pdi/dtf/teresa.cabre/docums/ca07arra.pdf; consulta: 13/07/2011].

CORDERO MONGE, SERGIO (2009): «Algunas consideraciones sobre el lenguaje común y el lenguaje técnico», Káñina, Revista de Artes y Letras de la Universidad de Costa Rica, XXXIII, pp. 75-80 [en línea en http://www.latindex.ucr.ac.cr/kanina-33-especiallex/kanina-33-especial-lex-07.pdf; consulta: 13/07/2011).

PORTO DAPENA, José-Álvaro (2002): Manual de técnica lexicográfica, Madrid, Arco/Libros.

, dir., Eugenia Conde Noguerol, Félix CóRdobA Rodríguez y M. ${ }^{a}$ Montserrat Muriano Rodríguez (2007): El Diccionario 'Coruña' de la lengua española actual: planta y muestra, Anexos de Revista de Lexicografía, 9, A Coruña, Universidade da Coruña.

RamíreZ, Nelson (2008): «Presentación», Acta Botánica Venezuelica 31/2, pp. 329-330 [en línea en http://www.scielo.org.ve/pdf/abv/v31n2/art02.pdf; consulta: 13/02/2011].

SANTAMARÍA PÉREZ, ISABEL (2006): «El léxico de la ciencia y de la técnica» [en línea en http://rua.ua.es/dspace/bitstream/10045/12767/8/L\%C3\%A9xico_de_la_ciencia_y_de_ la_t\%C3\%A9cnica.pdf; consulta: 07/07/2011].

TRAVESET, ANNA (2002): «Taller: Aspectos teóricos de la biología reproductiva de plantas y su relevancia para la conservación», Revista Chilena de Historia Natural, 75/1, pp. 3 4 [en línea en http://www.scielo.cl/pdf/rchnat/v75n1/art01.pdf; consulta: 22/2/2011]. 